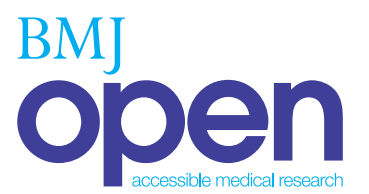

\title{
Early rehabilitation in critical care (eRiCC): functional electrical stimulation with cycling protocol for a randomised controlled trial
}

\author{
Selina M Parry, ${ }^{1,2}$ Sue Berney, ${ }^{2}$ René Koopman, ${ }^{3}$ Adam Bryant, ${ }^{1}$ Doa El-Ansary, \\ Zudin Puthucheary, ${ }^{4}$ Nicholas Hart, ${ }^{5}$ Stephen Warrillow, ${ }^{6}$ Linda Denehy ${ }^{1}$
}

To cite: Parry SM, Berney S, Koopman R, et al. Early rehabilitation in critical care (eRiCC): functional electrical stimulation with cycling protocol for a randomised controlled trial. BMJ Open 2012;2:e001891.

doi:10.1136/bmjopen-2012001891

- Prepublication history for this paper are available online. To view these files please visit the journal online (http://dx.doi.org/10.1136/ bmjopen-2012-001891).

Received 29 July 2012 Accepted 14 August 2012

This final article is available for use under the terms of the Creative Commons Attribution Non-Commercial 2.0 Licence; see http://bmjopen.bmj.com

For numbered affiliations see end of article.

\section{Correspondence to}

Selina Parry;

selina.parry@austin.org.au

\section{ABSTRACT}

Introduction: Intensive care-acquired weakness is a common problem, leads to significant impairment in physical functioning and muscle strength, and is prevalent in individuals with sepsis. Early rehabilitation has been shown to be safe and feasible; however, commencement is often delayed due to a patient's inability to co-operate. An intervention that begins early in an intensive care unit (ICU) admission without the need for patient volition may be beneficial in attenuating muscle wasting. The eRiCC (early rehabilitation in critical care) trial will investigate the effectiveness of functional electrical stimulation-assisted cycling and cycling alone, compared to standard care, in individuals with sepsis.

Methods and analysis: This is a single centre randomised controlled trial. Participants $(\mathrm{n}=80)$ aged $\geq 18$ years, with a diagnosis of sepsis or severe sepsis, who are expected to be mechanically ventilated for $\geq 48 \mathrm{~h}$ and remain in the intensive care $\geq 4$ days will be randomised within $72 \mathrm{~h}$ of admission to (1) standard care or (2) intervention where participants will receive functional electrical muscle stimulation-assisted supine cycling on one leg while the other leg undergoes cycling alone. Primary outcome measures include: muscle mass (quadriceps ultrasonography; bioelectrical impedance spectroscopy); muscle strength (Medical Research Council Scale; hand-held dynamometry) and physical function (Physical Function in Intensive Care Test; Functional Status Score in intensive care; 6 min walk test). Blinded outcome assessors will assess measures at baseline, weekly, at ICU discharge and acute hospital discharge. Secondary measures will be evaluated in a nested subgroup $(n=20)$ and will consist of biochemical/ histological analyses of collected muscle, urine and blood samples at baseline and at ICU discharge.

Ethics and dissemination: Ethics approval has been obtained from the relevant institution, and results will be published to inform clinical practice in the care of patients with sepsis to optimise rehabilitation and physical function outcomes.

Trial registration: Australian and New Zealand Clinical Trials Registry ACTRN12612000528853.

\section{ARTICLE SUMMARY}

Article focus

- Early rehabilitation is now advocated for individuals who are at risk of developing intensive care unit acquired weakness (ICUAW).

- Can FES(functional electrical stimulation)-assisted cycling or cycling alone minimise muscle mass and strength reductions, and improve discharge physical function, compared with standard care in patients with sepsis?

- What are the cellular and molecular mechanisms responsible for muscle changes in this patient population, and can these be attenuated using FES cycling or cycling alone?

Key messages

- This protocol outlines a randomized controlled trial (RCT) that will investigate the effectiveness of an FES-assisted cycling intervention and cycling alone, commencing within $72 \mathrm{~h}$ of ICU (intensive care unit) admission compared to standard care.

- The results of this trial will provide data to guide the early rehabilitation treatment of patients with sepsis.

- Muscle biopsies and biomarker analyses will provide insights into the effects of sepsis, and an intensive care admission, and early rehabilitation on intracellular signalling pathways and histochemical changes responsible for muscle mass losses.

Strengths and limitations of this study

- This is the first time that FES-assisted cycling has been investigated within the ICU within $72 \mathrm{~h}$ of admission with sepsis.

- This study combines bench-side research (biopsies and biomarker analyses) with patient-centred outcomes (strength and physical function).

- It is a single centre trial so results may need to be generalised with caution. 


\section{INTRODUCTION}

\section{Background}

Intensive care-acquired weakness (ICUAW) is a common problem following an ICU admission ${ }^{1-3}$ and is associated with prolonged hospitalisation, delayed weaning and increased mortality. ${ }^{4-6} \mathrm{Up}$ to $25 \%$ of patients requiring mechanical ventilation (MV) for greater than 7 days develop ICUAW, ${ }^{1}$ and this figure may rise to $50-100 \%$ in the septic population. ${ }^{78}$ Long-term follow-up studies of survivors of critical illness have demonstrated significantly impaired health-related quality of $\operatorname{life}^{9}{ }^{10}$ and physical functioning ${ }^{11-14}$ up to 5 years after ICU discharge, with weakness being the most commonly reported physical limitation. ${ }^{12}$ While survival has been a main focus of intensive care research, there is a paradigm shift to investigating methods to improve other patient-centred outcomes. ${ }^{15}$ There has been an increased awareness worldwide of the potential impact and benefit of early rehabilitation in the ICU. ${ }^{15-19}$ Early rehabilitation in the form of mobilisation has been shown to be safe and feasible $;^{20-24}$ however, it relies on the patient being co-operative, and to have sufficient cardiorespiratory reserve and medical stability ${ }^{25}$ to participate in therapy.

Muscle mass is known to reduce by at least $1.6 \%$ per day, ${ }^{26}$ with a $16-20 \%$ reduction in muscle mass within the first week in critically ill individuals with severe sepsis, ${ }^{27}$ indicating that interventions to attenuate muscle wasting in this initial stage may be beneficial. The musculoskeletal system is a highly plastic and adaptive system, responding quickly to changes in the demands placed upon it. ${ }^{3} 829$ The pathogenicity and molecular mechanisms for ICUAW have primarily been extrapolated from animal and in vitro muscle wasting models ${ }^{30-33}$ with ubiquitin-proteasome-mediated breakdown postulated to be primarily responsible for the muscle loss observed in critically ill individuals. ${ }^{30}{ }^{34-36}$ Local and systemic inflammatory processes, which occur in critically ill individuals, are thought to lead to a disruption in the balance between muscle protein synthesis and protein breakdown, leading to an overall reduction in muscle mass and force generation capacity. ${ }^{30}{ }^{37}$ Increased circulating inflammatory cytokines (eg, TNF- $\alpha$ and IL-1 $\beta$ ) may drive mitochondrial oxidative stress and increase intracellular calcium, which are postulated to trigger muscle proteolytic pathways ${ }^{30} 38$ and may interfere with insulin signalling leading to anabolic resistance, ${ }^{39}$ and contribute to electrophysiological inexcitability of the muscle ${ }^{40}$ Recent clinical trials in critically ill individuals have demonstrated a reduction in muscle myofibre size with preferential proteolysis of the thick myosin filaments, ${ }^{41} 42$ with one trial demonstrating a dramatic increase in protein degradation of up to $160 \% .{ }^{42}$ Currently, the pathogenesis of ICUAW is poorly understood given limited research within human clinical trials. ${ }^{2} 30$ Establishing the cellular and molecular mechanisms responsible for loss of muscle mass and strength is essential to help develop future medical and physical therapies.
There is growing interest in the use of assistive technologies to enable patients to commence therapy early in an ICU admission. ${ }^{43}$ Supine cycle ergometry, which can be utilised passively, actively (by patient effort) or active assisted (using electrical stimulation) ${ }^{16}$ has been studied in ICU within one trial with promising results. ${ }^{16}$ However, the intervention did not begin until at least 1-week post admission and there were no data reporting frequency of active versus passive cycling. ${ }^{16}$ Neuromuscular electrical stimulation (NMES) creates passive (ie, non-volitional) contraction of skeletal muscles through the use of low-voltage electrical impulses delivered through to the skin to underlying muscle via surface electrodes. ${ }^{43}$ It can be commenced early, without the need for patient participation and has been shown to prevent skeletal muscle atrophy in healthy individuals ${ }^{44}$ and improve physical function and strength in chronic disease populations, such as heart failure and chronic obstructive pulmonary disease. ${ }^{45}$ To date, studies within the ICU have involved stimulation of only isolated muscle groups such as the quadriceps, or peroneal muscles, in a resting non-functional position using NMES, with conflicting findings. ${ }^{27}{ }^{46-50}$ Further rigorous research needs to be conducted to determine the optimal stimulation settings, and efficacy of these interventions particularly post ICU on muscle strength and physical function, which is being investigated in one trial currently underway in the USA. ${ }^{51}$

Functional electrical stimulation (FES) is different to NMES, as it recruits muscles in functional patterns stimulating them in a similar way to how the muscles would 'normally' contract under volitional control in healthy individuals. For FES, the majority of the literature to date has been developed within the chronic stroke $^{52}$ and spinal cord injury (SCI) populations. ${ }^{53}$ Alternating recruitment of several muscle groups in a functional activity, such as cycling has been demonstrated in a chronic SCI population to improve the length of time a contraction can be sustained, prior to reaching the point of fatigue. ${ }^{54}$ This may enable patients to train for a longer period of time, thereby enhancing the training effect. FES-assisted cycling may influence muscle strength and physical function not only at ICU discharge, but also at acute hospital discharge. This trial seeks to examine the combined effect of FES-assisted cycling on muscle mass, strength, and physical function, and compare this with cycling alone, and standard care.

\section{Objectives}

The primary objectives are to:

1. Evaluate the effectiveness of FES-assisted cycling and cycling alone compared with standard care on muscle mass/cross-sectional area, strength and physical function, in individuals admitted to ICU with sepsis.

2. Evaluate the effectiveness of FES-assisted cycling compared with cycling alone, on muscle mass, muscle architecture, strength and physical function in individuals admitted to ICU with sepsis. 
The secondary objectives are to:

1. Establish the intracellular signalling pathways and histochemical changes responsible for muscle mass losses in individuals with sepsis.

2. Inform decisions about outcome measures and power calculations for future studies.

\section{Hypotheses for the primary aims}

H1. Patients who undergo FES-assisted cycling and cycling alone compared with standard care will have improved muscle mass/cross-sectional area, strength and physical function.

H2. Patients who undergo FES-assisted cycling when compared with cycling alone will have greater improvement in muscle mass, strength and physical function.

H3. Patients in the intervention group will have improved activity of anabolic signalling pathways when compared with patients receiving standard care as demonstrated from biomarker analysis.

\section{METHODS}

Trial design

This is an assessor blinded randomised controlled trial (RCT) which will be conducted at Austin Health in Melbourne, Australia.

\section{Participants}

Inclusion and exclusion criteria: Eighty participants in ICU (Austin Health, Melbourne, Australia) meeting the eligibility criteria will be recruited. Inclusion Criteria: (1) $\geq 18$ years, (2) expected $\mathrm{MV}>48 \mathrm{~h}$ with diagnosis of sepsis or severe sepsis as defined by ACCP Consensus Conference Criteria ${ }^{55}$ and (3) predicted ICU LOS $\geq 4$ days. Exclusion Criteria: (1) known primary systemic neuromuscular disease or intracranial process at admission; (2) lower limb amputation/s; (3) unable to perform study physical outcome measures premorbidly due to condition impairing mobility; (4) assessed by medical staff as approaching imminent death or withdrawal of medical treatment within $36 \mathrm{~h}$; (5) pregnancy; (6) $\mathrm{BMI}>40$; (7) presence of external fixator or superficial metal in lower limb; (8) open wounds or skin abrasions at electrode application points; (9) presence of pacemaker or implanted defibrillator; (10) transferred from another ICU after $>2$ days of consecutive MV; (11) platelets $<40000$ and INR $>1.6$ (for biomarker subgroup).

\section{Recruitment timeframe}

This study will take place in a single centre tertiary 20-bed ICU over an 18-month period. The ICU has a throughput of 2100 patients per year with 200 patients/ year who are both septic and requiring MV for $>48 \mathrm{~h}$.

\section{Recruitment and randomisation}

Patients meeting the above criteria will be invited to participate. Written informed consent from the patient, next of kin (NOK) or substitute decision maker will be sought. Participants will be asked to provide ongoing consent when able. Participants will be randomly assigned $[1: 1]$ to receive either standard care or the intervention using offsite-independent randomisation. Concealed allocation will be performed using sequentially numbered opaque sealed envelopes only accessible by research personnel with no involvement in the trial. Intervention arm: leg allocation will be randomly allocated $[1: 1]$ to FES-assisted cycling or cycling alone.

This trial involves a two-tier consent process first to the FES-assisted cycling intervention and then additionally to biomarker analysis and muscle biopsies with a nested subgroup $(n=20)$ within the primary trial. Details of all participants who refuse consent to muscle biopsy will be recorded. Information regarding screening and flow through this trial will be reported according to the CONSORT (Consolidated Standards of Reporting Trials) Extended Non-Drug guidelines. ${ }^{56}$ This trial has been approved by the Human Research Ethics Committee, Austin Health and registered with the Australian and New Zealand Clinical Trials Registry Identifier: ACTRN12612000528853.

\section{Procedure}

The planned flow of participants throughout the study is provided in figure 1. Once consent is obtained, and prior to randomisation; participants will be referred to a physiotherapist who will administer baseline testing of muscle mass/cross-sectional area (CSA) using diagnostic ultrasound (US) and body composition analysis. Once the patient is awake, a blinded outcome assessor will assess baseline strength and physical function once per week. 'Wakefulness' is defined as being able to follow $>3$ of 5 commands as assessed by the De Jonghe's readiness scale $^{1}$ with a Riker Sedation Agitation Scale (SAS) of 3-5. ${ }^{57}$ Biomarker assessment involving muscle biopsies, urine and blood analyses will be assessed at baseline and ICU discharge only. Attrition will be monitored and reasons for withdrawal will be recorded in each trial arm within figure 1 .

\section{Standard care}

Both groups will receive usual medical and nursing care in the ICU and ward settings. In the ICU, physiotherapy will be administered according to a standardised protocol, developed from a previous trial at the same institution. Patients in both arms will undergo usual care physiotherapy including respiratory and mobilisation/rehabilitation. ${ }^{20}$ Mobilisation activities (including those used for the benefit of the respiratory system) will be provided for up to but not more than $15 \mathrm{~min}$. Where possible the level and type of exercise delivered, will be prescribed using the initial PFIT results for each individual patient as per standard care. ${ }^{58}$ Details of physiotherapy treatment will be recorded but not protocolised on the acute hospital ward. 


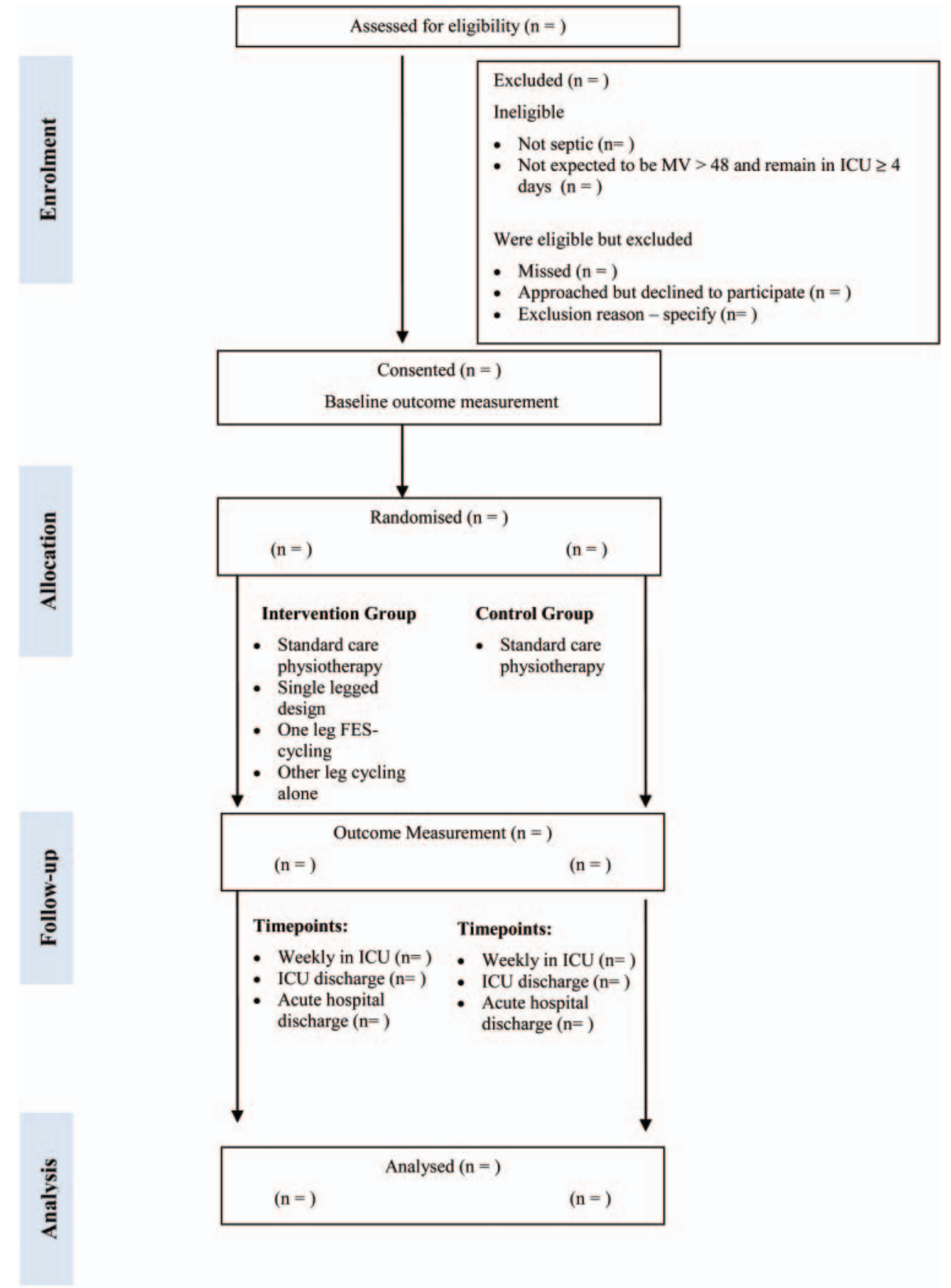

Figure 1 Consort diagram giving flow of participants throughout the study.

Abbreviations: FES-cycling, functional electrical stimulation-cycling; ICU, intensive care unit; MV, mechanical ventilation; $n$, number.

\section{Intervention}

The intervention will start within $72 \mathrm{~h}$ of ICU admission, continuing until ICU discharge. Participants will undertake up to $1 \mathrm{~h}$ of supine cycling daily, $\geq 5$ times weekly using a supine cycle ergometer (RT-300 supine model Restorative Therapies, Ltd, Baltimore, Maryland, USA) attached to a six-channel stimulator (SAGE stimulator, Restorative Therapies Ltd, Baltimore, Maryland, USA) and two RT50 wireless stimulator channels. Surface electrodes will be applied to the gluteal, hamstrings, quadriceps and calf muscles on both legs. However, the cycling only leg will not have the electrodes switched on (sham).
The intensity of muscle stimulation will be delivered at a level able to cause visible contractions (confirmed by palpation if uncertain) in all muscle groups without causing undue pain or discomfort to the participant. Pain levels will be closely monitored during and post each intervention session (box 1). If no contractions can be elicited this will be recorded, but the treatment continued, and palpation for contraction assessed at $5 \mathrm{~min}$ intervals. FES intensity will be gradually increased to a maximum of $140 \mathrm{~mA}$; with pulse duration of 300-400 $\mu \mathrm{s}$; frequency between 30 and $50 \mathrm{~Hz}$; and a pedal cadence between 30 and $45 \mathrm{rpm}$. Stimulation parameters will be 


\section{Box 1 Safety guidelines for exercise}

Safety guidelines: exercise should not be delivered or should be ceased when:

1. Patient mean arterial blood pressure $<65$ or below target pressure.

2. Patient heart rate is $<50$ or $>140$ beats/min or new arrhythmia develops (including ventricular ectopics or new onset atrial fibrillation).

3. Patient requires $>30 \mu \mathrm{g}$ of noradrenaline or comparable inotropic or vasopressor support.

4. Patient complains of new onset chest pain.

5. Patient becomes pale or sweaty and/or patient specifically requests to stop due to feeling acutely unwell.

6. Presence of extracorporeal membrane oxygenation or intraaortic balloon pump.

7. $\mathrm{FiO}_{2}>0.8$.

8. PEEP $>15 \mathrm{~cm} \mathrm{H}_{2} \mathrm{O}$.

9. Respiratory rate $>35$ breaths $/$ min sustained for $>60 \mathrm{~s}$.

10. $\mathrm{spO}_{2}$ falls $>10 \%$ below resting level or $<85 \%$ for $>60 \mathrm{~s}$.

11. Numerical rating scale (NRS) pain level remains $>7$ for 5 min despite adjusting stimulation intensity. Pain levels will be monitored using NRS if patient is awake at commencement and 5 mins into the exercise session. If the patient is intubated we will use standardised assessment of pain including facial grimacing, ventilator dysinchrony, and agitation levels to monitor pain levels during exercise.

$\mathrm{FiO}_{2}$, Fraction of inspired oxygen; $\mathrm{mm} \mathrm{Hg}$, millimetres of mercury; $\mu \mathrm{g}$, micrograms; NRS, Numerical Rating Scale; PEEP, Positive end expiratory pressure; $\mathrm{spO}_{2}$, saturation of peripheral oxygen.

adjusted to achieve the best possible muscle contraction for the patient. Once the patient is more alert, and able to participate, they will be provided with standardised encouragement to engage in therapy. To increase the intervention workload, resistance $(\mathrm{N} \mathrm{m})$ will be increased incrementally and cycling cadence up to a maximum of
$45 \mathrm{rpm}$. If a participant is readmitted to intensive care, the intervention will be re-instigated until discharge to the acute ward.

\section{Blinding}

While participants and intervention physiotherapist cannot be blinded to group allocation, outcome assessors and data analysts will be blinded to group allocation. The success of blinding will be assessed using a short outcome assessor questionnaire for each time point and the number of 'unblinding' episodes will be recorded. If an assessor becomes unblinded, an alternate assessor who is 'blinded' will continue to do the measures for that particular individual.

\section{OUTCOME MEASURES}

Table 1 provides a summary of the outcome measures and the time-points at which they will be assessed. All primary outcome measures will be measured at baseline; weekly while in ICU; at ICU discharge; and acute hospital discharge.

Baseline descriptive data collection will include age, gender, social, working and smoking history, admission diagnosis, hand/leg dominance, comorbidities as determined by the Functional Comorbidity Index and Charlson Comorbidity Index, severity of illness scoring and SAS Score. Additionally number of days of sedation and nutritional parameters, will also be recorded. ICU LOS, ICU free days at day 28, MV hours, tracheostomy requirement, incidence of ICU readmission, acute hospital LOS, discharge destination and mortality will also be recorded.

\section{PRIMARY OUTCOME MEASURES}

\section{Muscle mass}

1. Bioelectrical impedance spectroscopy (BIS): Participants will undergo measurement of body composition

Table 1 Summary of outcome measures and time points of assessment

\begin{tabular}{|c|c|c|c|c|}
\hline & Baseline & Weekly in ICU & ICU discharge & Acute hospital discharge ${ }^{\star \star}$ \\
\hline \multicolumn{5}{|c|}{ Muscle composition and thickness measures } \\
\hline Bioimpedance spectroscopy & $\checkmark$ & $\checkmark$ & $\checkmark$ & $\checkmark$ \\
\hline Quadriceps ultrasonography & $\checkmark$ & $\checkmark$ & $\checkmark$ & $\checkmark$ \\
\hline \multicolumn{5}{|l|}{ Strength measures } \\
\hline MRC score & $\checkmark$ & $\checkmark$ & $\checkmark$ & \\
\hline HHD grip and quadriceps & $\checkmark$ & $\checkmark$ & $\checkmark$ & $\checkmark$ \\
\hline \multicolumn{5}{|l|}{ Physical function measures } \\
\hline PFIT & $\checkmark$ & $\checkmark$ & $\checkmark$ & \\
\hline FSS-ICU score & $\checkmark$ & $\checkmark$ & $\checkmark$ & \\
\hline 6-MWT & & & & $\checkmark$ \\
\hline \multicolumn{5}{|l|}{ Biomarker measures } \\
\hline Muscle biopsy & $\checkmark$ & & $\checkmark$ & \\
\hline Urine analyses & $\checkmark$ & $\checkmark$ & $\checkmark$ & \\
\hline Blood analyses & $\checkmark$ & $\checkmark$ & $\checkmark$ & \\
\hline
\end{tabular}


using multi-frequency BIS machine (ImpediMed SFB7, ImpediMed Ltd, Brisbane, Australia). This will enable calculation of total body water, extracellular and intracellular fluid as well as fat-free mass, and an estimate of muscle mass. ${ }^{59}$ Measurements will be taken in triplicate with participants in the supine position after single use dual tab gel electrodes have been placed on one foot and one hand on the same side. ${ }^{60}$ All data will be uploaded onto the BioImped software (BIS) for subsequent analysis. The reliability of this device in both the critically ill and healthy populations has previously been established ${ }^{61}$ and numerous investigators have established the validity of the BIS in detecting within subjects change over time in septic and postsurgical populations. ${ }^{62} 63$

2. Diagnostic two-dimensional (2D) ultrasonography (US) of quadriceps muscle: Rectus Femoris and Vastus Intermedius CSA and thickness will be measured using diagnostic US (Voluson e BT09 Ultrasound, GE Healthcare, Yokogawa Medical Systems Ltd, Japan). Participants will lie in supine with their leg in passive extension and neutral rotation. A watersoluble transmission gel will be applied to the US head to allow acoustic contact, without depressing the dermal surface. The scanning head will be applied perpendicular to the long axis of the thigh on its anterior surface, approximately three-fifth distance from anterior superior iliac spine to the superior patellar border. ${ }^{64}$ The point will be marked for consistency of US probe location. Measures will be made in triplicate and averaged. The same operator who is experienced in the measurements will perform all US examinations. For supplementation of muscle thickness measurements, circumference of the thigh will be assessed using a tape measure at the US measurement site. Reliability and validity of ultrasonography have previously been established both in septic and other medical populations. ${ }^{65-67}$

\section{Muscle strength}

1. Medical Research Council Scale (MRC): manual muscle testing will be scored using the six point MRC scale. ${ }^{68}$

2. Hand-held dynamometry (HHD): Hand grip and quadriceps strength (supine with 5 -inch bolster under knee to enable flexion) ${ }^{69}$ will be assessed using HHD bilaterally using a Commander PowerTrack II Dynamometer 1500 (Banner Therapy Ltd, Asheville, North Carolina, USA) and a Jamar Dynamometer (Sammons Preston Rolyan, Bolingbrook, Illinois, USA), respectively. HHD has established reliability and validity. ${ }^{69} 70$

\section{Physical function}

Will be assessed using several different methods:

1. 'PFIT' (Physical Function Independence Test) ${ }^{71}$

2. 'FSS-ICU' (Functional Status Score in ICU) ${ }^{72}$

3. The 'six minute walk test' (6-MWT) is a commonly used test, which measures the distance that a patient can walk quickly in 6 min over a $25 \mathrm{~m}$ flat hallway. It is both self-paced and sub-maximal. The 6 MWT has previously been used in the ICU population. This test will only be performed at acute hospital discharge. ${ }^{73}$

\section{SECONDARY OUTCOME MEASURES \\ Biomarker analysis}

Participants who consent to participation will also be approached for consent to biomarker assessment involving muscle biopsies, blood and urine samples. Within the intervention arm as previously described, one leg undergoes cycling alone and the other FES-assisted cycling. Therefore, muscle biopsies will be collected from both lower limbs in the intervention arm, and in one lower limb in the standard care arm, to allow comparison across the groups. Biomarker assessment will be performed at baseline, and at ICU discharge (where possible the 'ICU discharge' measure will occur $24 \mathrm{~h}$ post final intervention exercise session).

\section{Muscle biopsy}

Using an aseptic technique, under local anaesthetic injected into the skin and fascia, muscle biopsies (100-200 mg of tissue) will be obtained from the vastus lateralis muscle, $10 \mathrm{~cm}$ above the patella. Prior to biopsies being performed, senior medical staff will review anticoagulation profiles, and using the Bergstrom percutaneous needle technique under suction ${ }^{74}$ will perform biopsies. Once the tissue has been extracted, part of the biopsy sample $(20-50 \mathrm{mg}$ ) will be dissected carefully, freed from any visible non-muscle material, embedded in Optimal Cutting Temperature (OCT) compound and immediately frozen in liquid nitrogen-cooled isopentane and stored at $-80^{\circ} \mathrm{C}$ until histochemical analyses. The remainder of the biopsy $(100-150 \mathrm{mg})$ will be immediately frozen in liquid nitrogen and stored at $-80^{\circ} \mathrm{C}$, until further analyses. Histological assessments of muscle structure and infiltration of inflammatory cells, will be performed using H\&E staining, and examination of muscle fibre-type-specific changes in cross sectional area, oxidative capacity, substrate stores, satellite cell activation and key proteins regulating protein synthesis and breakdown (mTOR, AMPK, MURF-1 and MAFbx). ${ }^{75}$ mRNA and protein expression levels and phosphorylation status of key signalling proteins in the regulation of inflammation will be determined using RT-PCR and standard immune-blotting techniques, respectively. ${ }^{76}$

\section{Urine analysis}

Twenty-four hour urine samples will be collected for analysis of urinary creatinine, 3-methylhistidine $(3-\mathrm{MH})$ and urea levels. The samples will be stored at $-80^{\circ} \mathrm{C}$ until analysis. 3-MH will be analysed using high-performance liquid chromatography (HPLC).

\section{Blood analysis}

Venous blood $(15 \mathrm{ml})$ will be collected at each time point and processed to obtain plasma and serum for 
subsequent analyses. Stored plasma and serum will be analysed using ELISA methods for IGF-1, IL-1 $\beta$, IL-6, IL-10, IL-18 and TNF- $\alpha$. Blood levels of C-reactive protein (CRP), urea and creatinine will be retrieved from the standard intensive care nursing charts over a 24-h period. HPLC will be performed to analyse vitamin D levels, serum amino acid concentration levels and 3-MH levels.

\section{SAFETY PROTOCOLS}

All physiotherapists providing intervention and outcome measurements are experienced ICU clinicians trained in safety and basic life support. Written manuals of protocols and safety guidelines will be made available to all research personnel. Criteria for commencing and ceasing exercise or outcome measurement are outlined in box 1.

If any adverse event occurs either during, or up to an hour following intervention or outcome measurement, the chief investigators will be notified. Serious adverse events are defined in this trial as 'adverse events', which results in death, cardiac arrest or stroke. These events will be reported to the Austin Health HREC. A data monitoring committee will examine trial safety by reviewing any adverse events at regular intervals. Research personnel to identify potential safety, recruitment, and treatment issues will review data process indicators based on the consort diagram monthly.

\section{STATISTICS}

\section{Sample size calculation}

The sample size calculation is informed by data from Burtin et $a l^{16}$ using the HHD improvement \pm SD for quadriceps strength of $0.54 \pm 0.76 \mathrm{~N} / \mathrm{kg}$. Using $\alpha=0.05$ and power $=0.80$ the total required sample size is 64 cases. Allowing for $14 \%$ in hospital mortality and dropouts the sample size has been increased to 80 cases. For biomarker analyses (primarily muscle biopsy) the sample size calculation is based on prior trials detecting changes in muscle protein synthesis and associated signalling following exercise, nutritional and pharmacological interventions (increase in signalling $>20 \%$ with SD in signalling of $2 \%) .^{77} \quad 78$ Using $\alpha=0.05$ and power $=0.80$ the required sample size is 18 cases. To account for dropouts and mortality we have increased this sample size to 20 cases.

\section{Statistical analysis}

All data will be entered into a purposefully designed database and exported to IBM SPSS Statistical software package (SPSS Inc, V.20, Chicago, Illinois, USA) for analyses. Descriptive statistics will first be used to examine the distribution for the two groups comparing baseline scores, to describe mean outcomes, and test whether parametric test assumptions have been met. Analyses will be by intention to treat. Statistical significance will be set at $\mathrm{p}<0.05$ (two-tailed). Possible differential attrition will be assessed by comparing baseline characteristics of those who withdraw or die against those who remain in the trial. Linear mixed modelling will be used to assess the treatment effects. Imputation of missing data will not be undertaken. Adjustment will be undertaken if significant imbalance is evident in baseline covariates and in this case results of both the adjusted and unadjusted results will be described. ${ }^{79}$ As a secondary analysis mean change in scores, 95\% CIs, and comparison with minimal clinically important differences in outcomes will be presented.

\section{ETHICS AND DISSEMINATION}

The Austin Health HREC has granted ethical approval for this trial. Trial results will be disseminated widely, through peer-review journal manuscripts and scientific conference presentations. The investigators will submit trial progress summaries to all sponsors of the trial on a regular basis.

\section{RESULTS}

The trial will determine whether FES-assisted cycling, or cycling alone compared to standard care will improve muscle mass, strength and physical function. The cellular and molecular mechanisms, responsible for the observed muscle mass and strength changes will be examined using biomarker analyses.

\section{DISCUSSION}

ICU-AW is a well-recognised clinical problem especially in individuals with sepsis. The effects of this syndrome have been shown to last for many years, with physical function one of the most affected patient outcomes. This research is innovative in that it combines both clinical and basic sciences to evaluate the mechanisms of change together with measuring patient-centred outcomes. It will be the first interventional trial to evaluate the effectiveness of FES-assisted cycling and cycling alone on muscle mass, architecture, strength and physical function. The important primary outcomes for the patient are expected to be improved or maintained strength, and physical function. Given the potentially devastating effects of a critical illness on the survivors and their families, ${ }^{15}$ gaining improved strength and physical function will be critical outcomes for all ICU survivors. The study design will allow future decisions to be made about sample sizes and primary outcomes to inform future research. The evaluation of an early rehabilitation programme involving FES-assisted cycling, which can commence in the critical period where the majority of muscle reductions occur, may provide direction for the development of appropriate rehabilitation in survivors of ICU.

\section{Author affiliations}

${ }^{1}$ Department of Physiotherapy, School of Health Sciences, The University of Melbourne, Melbourne, Australia

${ }^{2}$ Department of Physiotherapy, Austin Health, Melbourne, Australia 
${ }^{3}$ Department of Physiology, The University of Melbourne, Melbourne, Australia ${ }^{4}$ Department of Asthma, Allergy and Lung Biology, Institute of Health and Human Performance, University College London, Kings College London, London, UK

5Guy's and St Thomas' NHS Foundation Trust and King's College London, NIHR Comprehensive Biomedical Research Centre, London, UK

${ }^{6}$ Department of Intensive Care, Austin Health, Melbourne, Australia

Acknowledgements This research is being undertaken by SP as part of a doctoral qualification with the support of a National Health and Medical Research Council Dora Lush Scholarship (\#103923) and previously the Stella Mary Langford Scholarship. The authors would like to acknowledge the companies; Restorative Therapies and GE Healthcare for their support with training. The authors thank the staff of the physiotherapy and intensive care departments at Austin Health, Melbourne, Australia, for their ongoing support of the project.

Contributors All authors contributed to the study design, method and writing SP, LD, SB, RK, NH, AB and SW helped to develop the successful grant proposals, which support the study. SW and $\mathrm{NH}$ were involved in providing advice on safety; monitoring and ethical issues and LD will chair the data monitoring committee. SP, $\mathrm{AB}$ and $\mathrm{DE}$ contributed to the ultrasound protocol and analysis component of the trial. RK, ZP, NH and SW have specifically contributed to the biomarker analysis component of the trial. SP drafted the manuscript. All other authors critically revised it for important intellectual content. All authors contributed to and approved the final version of the manuscript for publication.

Funding Australian Intensive Care Foundation, Austin Medical Research Foundation and Society of Critical Care Medicine Vision Grant (United States) None of the funding bodies have any influence on the study design, collection or data analysis or publication of results.

Competing interests None.

Ethics approval Austin Health Research Ethics Committee.

Provenance and peer review Not commissioned; internally peer reviewed.

\section{REFERENCES}

1. De Jonghe $\mathrm{B}$, Sharshar $\mathrm{T}$, Lefaucheur J-P, et al. Paresis acquired in the intensive care unit-a prospective multicenter study. JAMA 2002;288:2859-67.

2. Puthucheary Z, Harridge S, Hart N. Skeletal muscle dysfunction in critical care: wasting, weakness and rehabilitation strategies. Crit Care Med 2010;38(Suppl):S676-82.

3. Puthucheary Z, Montgomery $\mathrm{H}$, Moxham J, et al. Structure to function: muscle failure in critically ill patients. J Physiol 2010;588:4641-8.

4. Ali NA, O'Brien JM, Hoffman SP, et al. Acquired weakness, handgrip strength, and mortality in critically ill patients. Am J Respir Crit Care Med 2008;178:261-8.

5. De Jonghe B, Bastuji-Garin S, Sharshar T, et al. Does ICU-acquired paresis lengthen weaning from mechanical ventilation? Intensive care med. Intensive Care Med 2004;30:1117-21.

6. Sharshar T, Bastuji-Garin S, Stevens RD, et al. Presence and severity of intensive care unit-acquired paresis at time of awakening are associated with increased intensive care unit and hospital mortality. Crit Care Med 2009;37:3047-53.

7. Tennila A, Salmi T, Pettila V, et al. Early signs of critical illness polyneuropathy in ICU patients with systemic inflammatory response syndrome or sepsis. Intensive Care Med 2000;26:1360-3.

8. Bolton CF. Sepsis and systemic inflammatory response syndrome: neuromuscular manifestations. Crit Care Med 1996;24:1408-16.

9. Adamson H, Elliott D. Quality of life after a critical illness: a review of general ICU studies 1998-2003. Aust Crit Care 2005;18:50-60.

10. Dowdy DW, Eid MP, Sedrakyan A, et al. Quality of life in adult survivors of critical illness: a systematic review of the literature. Intensive Care Med 2005;31:611-20.

11. Cheung AM, Tansey CM, Tomlinson G, et al. Two-year outcomes, health care use, and costs of survivors of acute respiratory distress syndrome. Am J Respir Crit Care Med 2006;174:538-44.

12. Herridge MS, Cheung AM, Tansey CM, et al. One-year outcomes in survivors of the acute respiratory distress syndrome. $N$ Engl J Med 2003;348:683-93.
13. Herridge MS, Tansey CM, Matté A, et al. Functional disability 5 years after acute respiratory distress syndrome. $N$ Engl J Med. 2011;364:1293-304.

14. Desai SV, Law TJ, Needham DM. Long-term complications of critical care. Crit Care Med 2011;39:371-9.

15. Needham DM, Davidson J, Cohen $\mathrm{H}$, et al. Improving long-term outcomes after discharge from intensive care unit: Report from a stakeholders' conference. Crit Care Med 2012;40:502-9.

16. Burtin C, Clerckx B, Robbeets $C$, et al. Early exercise in critically ill patients enhances short-term functional recovery. Crit Care Med 2009;37:2499-505

17. Kress JP. Clinical trials of early mobilization of critically ill patients. Crit Care Med 2009;37(Suppl):S442-7.

18. Truong $A D$, Fan E, Brower RG, et al. Bench-to-bedside review: mobilizing patients in the intensive care unit-from pathophysiology to clinical trials. Crit Care Med 2009;13:216-23.

19. Skinner EH, Berney S, Warrillow S, et al. Development of a physical function outcome measure (PFIT) and a pilot exercise training protocol for use in intensive care. Crit Care Med 2009;11:110-15.

20. Denehy L, Berney S, Skinner EH, et al. Evaluation of early rehabilitation for survivors of intensive care: protocol for a single blind randomised controlled trial. Open Crit Care Med J 2008;1: 39-47.

21. Morris PE, Goad A, Thompson C, et al. Early intensive care unit mobility therapy in the treatment of acute respiratory failure. Crit Care Med 2008;36:1-6.

22. Adler J, Malone D. Early mobilization in the intensive care unit: a systematic review. Cardiopulm Phys Ther J 2008;23:5-13.

23. Bailey P, Thomsen GE, Spuhler VJ, et al. Early activity is feasible and safe in respiratory failure patients. Crit Care Med 2007;35: 139-45.

24. Skinner EH, Berney S, Warrillow S, et al. Rehabilitation and exercise prescription in Australian Intensive Care Units. Physiotherapy 2008;94:220-9.

25. Gosselink R, Clerckx B, Robbeets C, et al. Physiotherapy in the intensive care unit. Netherlands J Crit Care 2011;15:66-75.

26. Reid CL, Campbell IT, Little RA. Muscle wasting and energy balance in critical illness. Clin Nutr 2004;23:273-80.

27. Poulsen JP, Møller K, Jensen CV, et al. Effect of transcutaneous electrical muscle stimulation on muscle volume in patients with septic shock. Crit Care Med 2011;39:456-61.

28. Flück M. Regulation of protein synthesis in skeletal muscle. Deutsche Zeitschrift Für Sportmedizin 2012;63:75-80.

29. Stewart CEH, Rittweger J. Adaptive processes in skeletal muscle: molecular regulators and genetic influences. J Musculoskelet Neuronal Interact 2006;6:73-86.

30. Bloch S, Polkey MI, Griffiths M, et al. Molecular mechanisms of intensive care unit-acquired weakness. Eur Respir J 2012;39: 1000-11.

31. Ochala J, Gustafson AM, Llano Diez M, et al. Preferential skeletal muscle myosin loss in response to mechanical silencing in a nove rat intensive care unit model: underlying mechanisms. J Physiol 2011;589:2007-26.

32. Ikemoto M, Nikawa T, Takeda S, et al. Space shuttle flight (STS-90) enhances degradation of rat myosin heavy chain in association with activation of ubiquitin-proteasome pathway. FASEB J 2001;15: 1279-81.

33. Gamrin L, Essen P, Hultman E, et al. Protein-sparing effect in skeletal muscle of growth hormone treatment in critically ill patients. Ann Surg 2000;231:577-86.

34. Tiao G, Hobler S, Wang JJ, et al. Sepsis is associated with increased mRNAs of the ubiquitin-proteasome proteolytic pathway in human skeletal muscle. J Clin Invest 1997;99:163-8.

35. Lecker $\mathrm{SH}$. Ubiquitin-protein ligases in muscle wasting: multiple parallel pathways? Curr Opin Clin Nutr Metab Care 2003;6:271-5.

36. Lecker SH, Jagoe RT, Gilbert A, et al. Multiple types of skeleta muscle atrophy involve a common program of changes in gene expression. FASEB J 2004;18:39-51.

37. Khan J, Harrison T, Rich M. Mechanisms of neuromuscular dysfunction in critical illness. Crit Care Clin 2008;24:165-77.

38. Shang F, Gong X, Taylor A. Activity of ubiquitin-dependent pathway in response to oxidative stress. Ubiquitin-activating enzyme is transiently up-regulated. J Biol Chem 1997;272:23086-93.

39. Rennie MJ. Anabolic resistance in critically ill patients. Crit Care Med 2009;379(Suppl):S398-9.

40. Z'Graggen W, Lin C, Howard R, et al. Nerve excitability changes in critical illness polyneuropathy. Brain 2006;129:2461-70.

41. Derde S, Hermans G, Derese I, et al. Muscle atrophy and preferential loss of myosin in prolonged critically ill patients. Crit Care Med 2012;40:79-89. 
42. Klaude $\mathrm{M}$, Mori M, Tjäder I, et al. Protein metabolism and gene expression in skeletal muscle of critically ill patients with sepsis. Clin Sci 2012;122:133-42.

43. Needham DM, Truong AD, Fan E. Technology to enhance physical rehabilitation of critically ill patients. Crit Care Med 2009;37(Suppl): S436-41.

44. Gibson JNA, Rennie MJ, Smith K. Prevention of disuse muscle atrophy by means of electrical stimulation: maintenance of protein synthesis. Lancet 1988;2:767-70.

45. Sillen MJH, Speksnijder CM, Eterman RMA, et al. Effects of neuromuscular electrical stimulation of muscles of ambulation in patients with chronic heart failure or COPD-asystematic review of the English-language literature. CHEST 2009;136:44-61.

46. Gruther W, Kainberger F, Fialka-Moser V, et al. Effects of neuromuscular electrical stimulation on muscle layer thickness of knee extensor muscles in intensive care unit patients: a pilot study. $J$ Rehabil Med 2010;42:593-7.

47. Karatzanos E, Gerovasili V, Zervakis D, et al. Electrical muscle stimulation: an effective form of exercise and early mobilization to preserve muscle strength in critically ill patients. Crit Care Res Pract 2012;1:1-8.

48. Routsi C, Gerovasili V, Vasileiadis I, et al. Electrical muscle stimulation prevents critical illness polyneuromyopathy: a randomized parallel intervention trial. Crit Care 2010;14:R74.

49. Gerovasili V, Stefanidis K, Vitzilaios K, et al. EmsptmmociparsCCElectrical muscle stimulation preserves the muscle mass of critically ill patients: a randomized study. Crit Care 2009;13:161-8.

50. Rodriguez PO, Setten M, Maskin LP, et al. Muscle weakness in septic patients requiring mechanical ventilation: protective effect of transcutaneous neuromuscular electrical stimulation. J Crit Care 2012;27:319e1-8.

51. Kho ME, Truong AD, Brower RG, et al. Neuromuscular Electrical Stimulation for Intensive Care Unit-acquired weakness: protocol and methodological implications for a randomized, Sham-controlled, phase II trial. Phys Ther 2012;March 15(Epub ahead of print).

52. Robbins SM, Houghton PE, Woodbury MG, et al. The therapeutic effect of functional and transcutaneous electrical stimulation on improving gait speed in stroke patients: a meta-analysis. Arch Phys Med Rehabil 2006;87:853-9.

53. Hamzaid NA, Davis GM. Health and fitness benefits of functional electrical stimulation-evoked leg exercises for spinal cord injured individuals: a position review top spinal cord. Inj Rehabil 2009;14:88-121.

54. Decker MJ, Griffin L, Abraham LD, et al. Alternating stimulation of synergistic muscles during functional electrical stimulation cycling improves endurance in persons with spinal cord injury. J Electromyogr Kinesiol 2010;20:1163-9.

55. Levy MM. 2001 SCCM/ESICM/ACCP/ATS/SIS International Sepsis Definitions Conference. Crit Care Med 2003;31:1250-6.

56. Boutron I, Moher D, Altman DG, et al. Methods and processes of the CONSORT group: example of an extension for trials assessing nonpharmacological treatment. Ann Intern Med 2008;148:60-6.

57. Fraser GL, Riker R. Monitoring sedation, agitation, analgesia and delirium in critically ill adult patients. Crit Care Clin 2001;17:1-21.

58. Berney S, Haines K, Skinner $\mathrm{EH}$, et al. The safety and feasibility of an exercise prescription approach to rehabilitation across the continuum of care for survivors of critical illness. Phys Ther 2012, in press.

59. Barbosa-Silva MCG. Bioelectrical impedance analysis in clinical practice: a new perspective on its use beyond body composition equations. Curr Opin Clin Nutr Metab Care 2005;8:311-17.

60. Lukaski HC, Bolonchuk WW, Hall CB, et al. Validation of tetrapolar bioelectrical impedance method to assess human body composition. J Appl Physiol 1986;60:1327-32.
61. Baldwin CE, Paratz JD, Bersten AD. Body composition analysis in critically ill survivors: a comparison of bioelectrical impedance spectroscopy devices. JPEN 2012;36:306-15.

62. Earthman C, Traughber D, Dobratz J, et al. Bioimpedance spectroscopy for clinical assessment of fluid distribution and body cell mass. Nutr Clin Practice 2007;22:389-405.

63. Frankenfield DC. Bioelectrical impedance plethysmographic analysis of body composition in critically injured and healthy subjects. $A m \mathrm{~J}$ Clin Nutr 1999;69:426-31.

64. Seymour JM, Ward K, Sidhu PS, et al. Ultrasound measurement of rectus femoris cross-sectional area and the relationship with quadriceps strength in COPD. Thorax 2009;64:418-23.

65. Baldwin CE, Paratz JD, Bersten AD. Diaphragm and peripheral muscle thickness on ultrasound: intra-rater reliability and variability of a methodology using non-standard recumbent positions. Respirology 2011;16:1136-43.

66. Campbell IT, Watt T, Withers D, et al. Muscle thickness measured with ultrasound may be an indicator of lean tissue wasting in multiple organ failure in the presence of oedema. Am J Clin Nutr 1995;62:533-9.

67. Ishida Y, Carroll JF, Pollock ML, et al. Reliability of B-mode ultrasound for the measurement of body fat and muscle thickness. Am J Hum Biol 1992;4:511-20.

68. Kleyweg RP. Inter-observer agreement in the assessment of muscle strength and functional abilities in Guillain-Barre syndrome. Muscle Nerve 1991;14:1103-9.

69. Baldwin CE, Paratz JD, Bersten AD. Muscle strength assessment in critically ill patients with handheld dynamometry: an investigation of reliability, minimal detectable change, and time to peak force generation. J Crit Care 2012;Article in Press.

70. Vanpee $\mathrm{G}$, Segers J, Van Mechelen $\mathrm{H}$, et al. The interobserver agreement of handheld dynamometry for muscle strength assessment in critically ill patients. Crit Care Med 2011;39:1929-34.

71. Denehy L, Skinner EH, Edbrooke L, et al., eds. A physical function test for use in the ICU: validity, responsiveness and predictive utility of the PFIT (scored). American Thoracic Society, Denver, 2011. Am $J$ Respir Crit Care Med 2011:A2642.

72. Zanni HM, Korupolu R, Fan E, et al. Rehabilitation therapy and outcomes in acute respiratory failure: an observational pilot project. J Crit Care 2010;25:254-62.

73. American Thoracic Society. ATS statement: guidelines for the Six-Minute Walk Test. Am J Respir Crit Care Med 2002;166:111-17.

74. Bergstrom J. Percutaneous needle biopsy of skeletal muscle in physiological and clinical research. Scand J Clin Lab Invest 1975;35:609-16.

75. Koopman R, Zorenc AH, Gransier RJ, et al. The increase in S6K1 phosphorylation in human skeletal muscle following resistance exercise occurs mainly in type II muscle fibers. Am J Physiol Endocrinol Metab 2006;290:E1245-52.

76. Koopman R,, Gehrig SM, Léger B, et al. Cellular mechanisms underlying temporal changes in skeletal muscle protein synthesis and breakdown during chronic \{beta\}-adrenoceptor stimulation in mice. J Physiol 2010;1(588, Pt 23):4811-23.

77. Pennings B, Koopman R, Beelen M, et al. Exercising prior to protein intake forms an effective strategy to stimulate post-prandial muscle protein synthesis in both young and elderly men. Am J Clin Nutr 2011;93:322-31.

78. Koopman R, Walrand S, Beelen M, et al. Dietary protein digestation and absorption rate and the subsequent muscle protein synthetic response are not different between young and elderly men. J Nutr 2009;139:1707-13.

79. Yu LM, Chan AW, Hopewell S, et al. Reporting on covariate adjustment in randomised controlled trials before and after revision of the 2001 CONSORT statement: a literature review. Trials 2010;11:59. 